\title{
Influence of heterologous MreB proteins on cell morphology of Bacillus subtilis
}

\author{
Correspondence \\ Jeff Errington \\ jeff.errington@newcastle.ac.uk
}

Received 14 May 2009

Revised 17 July 2009

Accepted 23 July 2009

\section{Kathrin Schirner and Jeff Errington}

\author{
Institute for Cell and Molecular Biosciences, Newcastle University, Medical School, Framlington \\ Place, Newcastle Upon Tyne NE2 4HH, UK
}

\begin{abstract}
The prokaryotic cytoskeletal protein MreB is thought to govern cell shape by positioning the cell wall synthetic apparatus at growth sites in the cell. In rod-shaped bacteria it forms helical filaments that run around the periphery of the rod during elongation. Gram-positive bacteria often contain more than one $m r e B$ gene. Bacillus subtilis has three $m r e B$-like genes, $m r e B, m b /$ and $m r e B H$, the first two of which have been shown to be essential under normal growth conditions.

Expression of an $m r e B$ homologue from the closely related organism Bacillus licheniformis did not have any effect on cell growth or morphology. In contrast, expression of mre $B$ from the phylogenetically more distant bacterium Clostridium perfringens produced shape defects and ultimately cell death, due to disruption of the endogenous MreB cytoskeleton. However, expression of either $m r e B^{B \text {. licheniformis }}\left(m r e B^{B /}\right.$ ) or $m r e B^{C \text {. perfringens }}$ ( $m r e B^{C p}$ ) was sufficient to confer a rod shape to $B$. subtilis deleted for the three $m r e B$ isologues, supporting the idea that the three proteins have largely redundant functions in cell morphogenesis. Expression of $m r e B C D^{B /}$ could fully compensate for the loss of $m r e B C D$ in $B$. subtilis and led to the formation of rodshaped cells. In contrast, expression of $m r e B C D^{C p}$ was not sufficient to confer a rod shape to $B$. subtilis $\triangle m r e B C D$, indicating that a complex of these three cell shape determinants is not enough for cell morphogenesis of $B$. subtilis.
\end{abstract}

\section{INTRODUCTION}

For a long time, the presence of cytoskeletal elements has been considered a characteristic eukaryotic trait. However, in the last decade it has emerged that prokaryotes also have proteins with cytoskeletal function. One large group of cytoskeletal elements are the actin homologues, represented mainly by the MreB proteins in bacteria. Homologues of MreB have been found in most species with complex, i.e. non-spherical, cell morphology (Bork et al., 1992; Carballido-López, 2006b; van den Ent et al., 2001). Gram-negative organisms tend to have one $m r e B$ gene, usually in an operon with $m r e C$ and $m r e D$ genes, whilst Gram-positive bacteria often have multiple mreB isologues, but only one of these normally occurs in an operon with mreCD.

Bacillus subtilis encodes three actin-like proteins called MreB, MreBH and Mbl. The $m r e B$ gene is defined by its position in an operon with $m r e C$ and $m r e D$, whilst the other two genes are organized in monocistronic units. The single mutants of any of the three mreB genes are viable if cultured in medium supplemented with high concentrations of $\mathrm{Mg}^{2+}$ : for $m r e B$ and $m b l$ mutants, a concentration of approximately $3 \mathrm{mM}$ is required, but the $\mathrm{mreBH}$ mutant

Abbreviations: NA, nutrient agar; PAB, Difco Antibiotic Medium 3; YFP, yellow fluorescent protein. only needs $100 \mu \mathrm{M} \mathrm{Mg}^{2+}$ to grow (Carballido-López et al., 2006; Formstone \& Errington, 2005; Schirner \& Errington, 2009). All three single mutants have a defect in cell morphology. mreB mutant cells have an increased diameter and are straight, whereas $m b l$ mutants are also wider but are characteristically twisted. $m r e B H$ mutant cells are straight but thinner than the wild-type. MreB, Mbl and MreBH co-localize, forming one helical structure along the long axis of the cell underneath the cytoplasmic membrane (Carballido-López et al., 2006; Jones et al., 2001). It has been suggested that they have a role in cell shape determination by organizing the peptidoglycan synthetic machinery at the cell surface (Carballido-López, 2006). This is consistent with observations that peptidoglycan synthesis occurs in a similar helical pattern (Daniel \& Errington, 2003; Hayhurst et al., 2008; Tiyanont et al., 2006a; Kawai et al., 2009a). MreC and MreD have a role in cell elongation, and interaction studies suggest that they are in the same morphogenic pathway as MreB (Figge et al., 2004; Jones et al., 2001; Kruse et al., 2005; Leaver \& Errington, 2005; Lee \& Stewart, 2003; Rogers et al., 1970; Wachi et al., 1989). Viability of $m r e C$ and $m r e D$ deletion strains is restricted to conditions in which the growth medium is supplemented with high concentrations of $\mathrm{Mg}^{2+}$, similar to mreB mutants, but additionally these strains require an osmoprotectant, such as sucrose, to prevent cell lysis; the mutant cells completely lose their rod shape and grow as 
clusters of spheres (Leaver \& Errington, 2005). A similar phenotype has been observed for a mutant deleted for the whole $m r e B C D$ operon (M. Leaver, unpublished data).

The current model suggests that the MreB helix is responsible for organizing the peptidoglycan synthetic apparatus, at least in part by interacting with membrane proteins, including MreC, MreD, RodA and one or more penicillin-binding proteins. MreBH has additionally been shown to be involved in the export of LytE, a cell wall hydrolase. By coordinating synthetic and lytic enzymes in one complex, the cell maintains the delicate balance between synthesis and hydrolysis of cell wall material (Carballido-Lopez, 2006a, b; Stewart, 2005).

Here we show that the three MreB-like proteins in $B$. subtilis have overlapping functions. Expression of one heterologous MreB protein from the closely related organism Bacillus licheniformis or the phylogenetically more distant organism Clostridium perfringens is enough for the formation of a long cell axis in a B. subtilis strain lacking all three endogenous MreB proteins. Interestingly, the presence of $\mathrm{MreB}^{\mathrm{C} \text {. perfringens }}\left(\mathrm{MreB}^{\mathrm{CP}}\right)$ has a dominantnegative effect on B. subtilis wild-type cells, and leads to cell morphology defects and loss of viability by interfering with the localization of the endogenous MreB proteins.

\section{METHODS}

General methods. All strains were grown on nutrient agar (NA; Oxoid) plates or as liquid cultures whilst shaking in Difco Antibiotic Medium 3 (PAB) at $37{ }^{\circ} \mathrm{C}$. Growth media were supplemented with $20 \mathrm{mM} \mathrm{Mg}^{2+}$ (added in the form of $\mathrm{MgSO}_{4}$ ) and/or xylose at the concentrations indicated. Growth curves were obtained by measuring optical density at $600 \mathrm{~nm}$ at the time points indicated.

DNA manipulations and Escherichia coli DH5 $\alpha$ transformations were carried out using standard methods (Sambrook et al., 1989). B. subtilis strains were transformed according to the method of Anagnostopoulos \& Spizizen (1961) as modified by Jenkinson (1983). Selection for B. subtilis transformants was carried out on NA plates, supplemented with the following antibiotics, as required $\left(\mu \mathrm{g} \mathrm{ml}^{-1}\right)$ : kanamycin (5), chloramphenicol (5), erythromycin (1), lincomycin (25) and/or spectinomycin (50).

Strain construction. All strains and plasmids used are listed in Table 1. To construct these strains, the $m r e B$ or $m r e B C D$ genes, respectively, were amplified from chromosomal DNA of B. licheniformis or C. perfringens with the oligonucleotides listed in Table 2 . The PCR products were ligated into plasmid pJPR1, placing the gene(s) under control of the xylose inducible promoter between two segments of the amyE gene. For construction of strain 4630, primers amy1 and amy2 were used to amplify the region to be inserted in the chromosome, and B. subtilis 168 was transformed directly with the PCR product. Plasmids pSG5934, pSG5935 and pSG5936 were cloned using E. coli DH5 $\alpha$. Transformation of $B$. subtilis with these plasmids or the linear PCR product and selection for chloramphenicol resistance resulted in strains carrying the heterologous gene(s) under control of the xylose inducible promoter inserted into the chromosome at the $a m y E$ locus. If required, deletions of endogenous genes were introduced afterwards.

Microscopic imaging. Cell morphology was assessed using light microscopy. Cells were grown on NA plates or in PAB with supplements as indicated at $37{ }^{\circ} \mathrm{C}$. Samples of exponentially grown cells were taken after diluting back once in the presence of inducer, and then mounted on microscope slides covered with a thin film of $1 \%$ agarose in minimal medium (Glaser et al., 1997). For fluorescence microscopy, overnight cultures were diluted into $\mathrm{CH}$ medium (Nicholson \& Setlow, 1990) and grown for $2 \mathrm{~h}$ at $37{ }^{\circ} \mathrm{C}$ in the presence of $0.5 \mathrm{mM}$ IPTG to induce expression of the fluorescent proteins. Expression of the heterologous $\mathrm{MreB}$ was induced by addition of $0.5 \%$ xylose, and the strains were grown for $1 \mathrm{~h}$ before taking samples for microscopy. Images were acquired with a Sony CoolSnap HQ cooled CCD camera (Roper Scientific) camera attached to a Zeiss Axiovert M135 microscope or to a Zeiss Axiovert M200 microscope. ImageJ (http://rsb.info.nih.gov/ij/) was used to analyse the images. Manipulation was limited to altering brightness and contrast to obtain optimal prints.

\section{RESULTS}

\section{Construction of strains expressing heterologous MreB proteins}

Under typical bacterial culture conditions, the $m r e B$ gene is essential to B. subtilis, but viability can be restored by addition of high concentrations of $\mathrm{Mg}^{2+}$ to the growth medium. When $\mathrm{Mg}^{2+}$ is removed, the cells start to swell and lyse (Formstone \& Errington, 2005). To investigate if expression of $m r e B$ from other bacteria could complement this defect, strains were constructed carrying the mreB genes amplified by PCR from B. licheniformis $\left(\mathrm{mreB}^{B l}\right)$ or C. perfringens $\left(m r e B^{C P}\right)$, then placed under the control of a xylose-inducible promoter at the $a m y E$ locus. Additionally, constructs were made in which the whole $m r e B C D$ operon of $B$. licheniformis or $C$. perfringens was placed under the control of $P_{\mathrm{xyl}}$ at the amyE locus.

The heterologous $m r e B$ genes were identified by BLAST searches (Altschul et al., 1990), and neighbourhood to paralogues of $m r e C D$ was used as a criterion to differentiate between $m r e B$ and endogenous orthologues. As in $B$. subtilis, the genome of $B$. licheniformis encodes three actin homologues (designated mreB, $m b l$ and $m r e B H$ ); in contrast, only two mreB-like genes were found in $C$. perfringens (designated $m r e B$ and $m b l$ ).

$\mathrm{MreB}^{B l}$ is $92 \%$ identical to $\mathrm{MreB}$ of B. subtilis $\left(\mathrm{MreB}^{B s}\right)$, whilst $\mathrm{MreB}$ of the less closely related organism $C$. perfringens is $63 \%$ identical to the $B$. subtilis protein. While MreC and MreD from B. licheniformis are 71 and $63 \%$ identical, respectively, to the B. subtilis proteins, their homologues in C. perfringens share only 21 and $16 \%$ identity, respectively. Table 3 summarizes the similarities of the MreB-like proteins of B. licheniformis and C. perfringens compared to their respective homologue in B. subtilis as determined by CLUSTAL W version 2.0 analysis (Larkin et al., 2007).

\section{Dominant-negative effect of $m r e B^{C p}$ expression}

No effect was observed when expression of $\mathrm{MreB}^{B l}$ was induced with $0.5 \%$ xylose in a B. subtilis 168 wild-type 
Table 1. Strains and plasmids

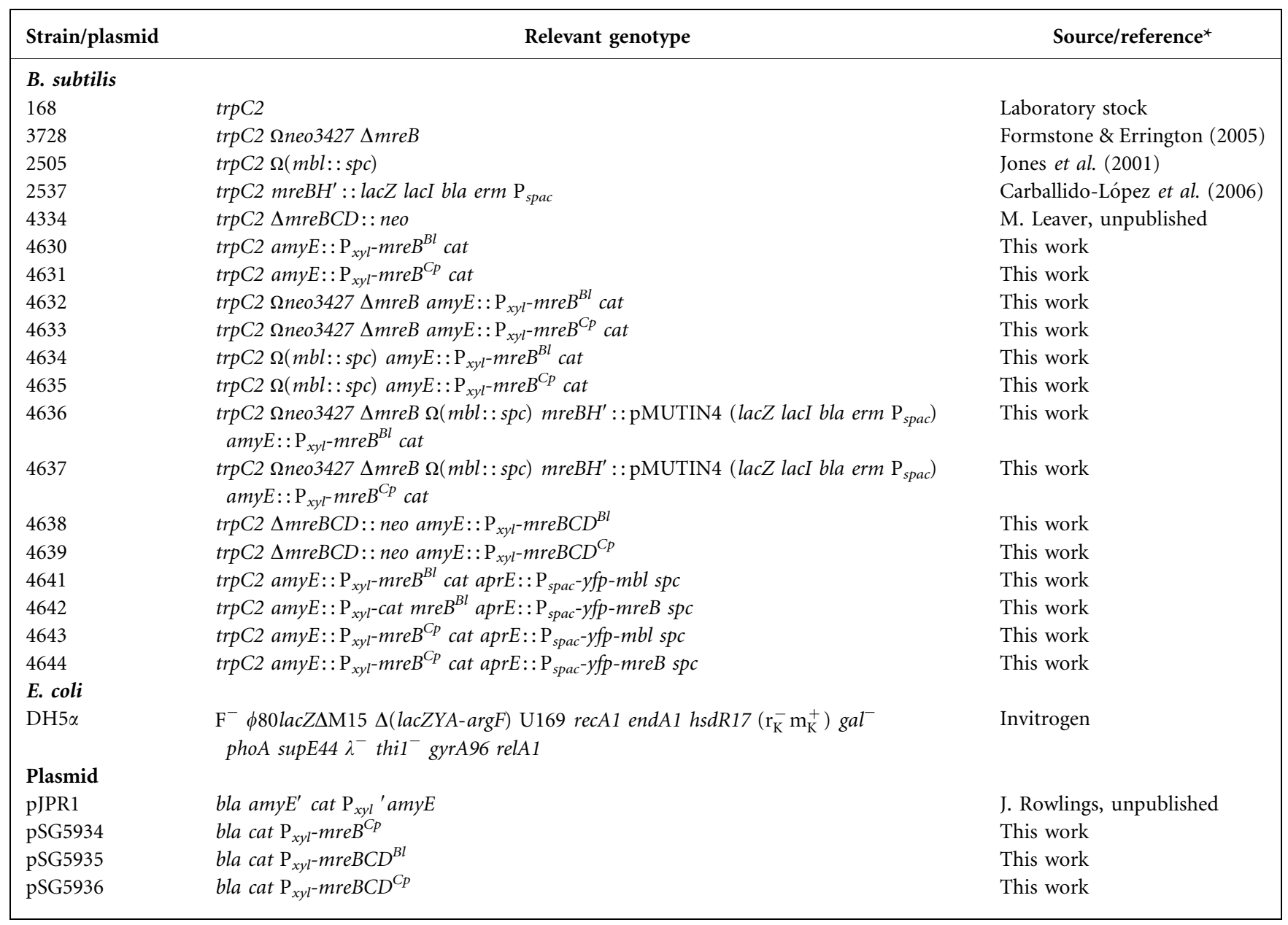

${ }^{*}$ M. Leaver, Institute for Cell and Molecular Biosciences, Newcastle University; J. Rowlings, c/o Sir William Dunn School of Pathology, University of Oxford.

background strain (4630). Growth on NA plates or in liquid $\mathrm{PAB}$ was not affected in comparison to the behaviour of an uninduced strain (Fig. 1a). Also, cell morphology was not altered by expression of $\mathrm{mreB}^{\mathrm{Bl}}$ (Fig. 1b). This result is consistent with observations made for overexpression of $g f p-m r e B^{B s}, g f p-m b l^{B s}$ or $g f p-m r e B H^{B s}$ under the same conditions (Carballido-López et al., 2006; Formstone \& Errington, 2005; Jones et al., 2001). Similarly, expression of $m r e B C D^{B l}$ did not have obvious effects on viability or cell morphology (data not shown).

In contrast, induction of $m r e B^{C p}$ expression had a deleterious effect on $B$. subtilis. Hardly any growth was observed on NA plates containing $0.5 \%$ xylose (strain 4631; Fig. 1a), and in liquid medium the growth rate decreased rapidly upon addition of inducer (data not shown). Microscopic examination revealed that induction of $m r e B^{C p}$ expression led to severe morphological defects: twisted cells, formation of chains and lysis (Fig. 1b, c). This effect was correlated with the amount of xylose added, and we therefore concluded that it was due to $m r e B^{C p}$ expression levels (Fig. 1c). The cell morphology was reminiscent of an $\mathrm{mbl}$ mutant of $B$. subtilis (Abhayawardhane \& Stewart, 1995; Jones et al., 2001; Schirner \& Errington, 2009), and similar to that strain, addition of $\mathrm{Mg}^{2+}$ restored viability (Fig. 1a) and ameliorated the cell shape defects (Fig. 1b). Comparable observations were made when the three genes $m r e B C D^{C p}$ were expressed in a wild-type background (data not shown).

\section{MreB $^{C p}$ disrupts the endogenous cytoskeleton}

To test whether expression of $m r e B^{C p}$ interfered with assembly of the MreB cytoskeleton of B. subtilis, we analysed the localization of YFP-Mbl and YFP-MreB in strains expressing heterologous $m r e B$ genes. In the absence of heterologous $m r e B$ genes, both YFP-Mbl and YFP-MreB localized along the periphery of the cells in a pattern that is thought to be based on an essentially helical structure [Fig. $2 \mathrm{a}(\mathrm{i}), \mathrm{b}(\mathrm{i}), \mathrm{c}(\mathrm{i}), \mathrm{d}(\mathrm{i})$ ]. Expression of $m r e B^{B l}$ did not change 
Table 2. Oligonucleotides used in this study

\begin{tabular}{|lcll|}
\hline Name & Restriction site & Sequence $\left(\mathbf{5}^{\prime}-\mathbf{3}^{\prime}\right)$ & \multicolumn{1}{c|}{ Purpose } \\
\hline lichB-fwEco & EcoRI & ATCGTAGAATTCAATTTATGGGTTTTTGCTTAGG & Amplification of $m r e B^{B l}$ \\
lichB-revBam & Bam $\mathrm{HI}$ & TCACATGGATCCTGCGGCATGATTATACAC & Amplification of $m r e B^{B}$ \\
lichD-revBam & Bam $\mathrm{HI}$ & GAGTTGGGATCCCTCTTTTCGACATCGGC & Amplification of $m r e B C D^{B l}$ \\
ClosperB-fw & SalI & ATCAGAGTCGACGAGACCCAACACCTAGTAGAG & Amplification of $m r e B^{C p}$ \\
ClosperB-rev & Bam $\mathrm{HI}$ & TAGTGAGGATCCGGCAACTGACAGTACTATAACAG & Amplification of $m r e B^{C p}$ \\
ClosperD-rev & Bam $\mathrm{HI}$ & ACACTCGGATCCTTGAACTTCTCATGTAACCACC & Amplification of $m r e B C D^{C p}$ \\
amy1 & & CCGTCTAGACTAGTTAAACGGCGAACAAATCGAAT & Amplification of insert from pJPR1 \\
amy2 & & CCCTCTAGACTAGTTTAAGCACTCCCGCGATCGCC & Amplification of insert from pJPR1 \\
\hline
\end{tabular}

this localization pattern [Fig. 2a(ii), b(ii)]. In contrast, when $m r e B^{C P}$ was expressed, the localization of both YFP$\mathrm{Mbl}$ [Fig. 2c(ii)] and YFP-MreB [Fig. 2d(ii)] was severely disrupted. The pattern of tilted bands was completely disrupted and replaced with small numbers of bright foci that appear cytosolic rather than peripheral. This disruption of the endogenous MreB cytoskeleton provides a clear explanation for the dominant-negative phenotype on expression of $m r e B^{C P}$.

\section{mre $B^{B I}$ but not $m r e B^{C p}$ can compensate for the loss of $B$. subtilis mreB}

To test if the heterologous MreB proteins could substitute for B. subtilis MreB, the $m r e B$ gene was deleted in strains harbouring the heterologous $m r e B$ genes $(4632,4633)$. When expression of $m r e B^{B l}$ was induced, viability of the otherwise inviable $B$. subtilis $\Delta m r e B$ strain was restored both on plates and in liquid culture without elevated $\mathrm{Mg}^{2+}$ concentrations (strain 4632; Fig. 3a, c). The formation of small colonies of strain 4632 on NA plates without supplements was probably due to incomplete repression of the xylose-inducible promoter. Although expression of $m r e B^{B l}$ could partially restore growth, cell morphology was still impaired. The cells often appeared bent, but lysis was not apparent (Fig. 3b).

Table 3. Relative conservation of the $\mathrm{MreB}, \mathrm{Mbl}$ and $\mathrm{MreBH}$ proteins

\begin{tabular}{|lcccc|}
\hline Source & Protein & \multicolumn{2}{c|}{$\begin{array}{c}\text { Percentage identity relative } \\
\text { to the } \text { B. subtilis protein }\end{array}$} \\
\cline { 3 - 5 } & & MreB & Mbl & MreBH \\
\hline B. subtilis & MreB & 100 & 53 & 56 \\
& $\mathrm{Mbl}$ & 53 & 100 & 51 \\
& $\mathrm{MreBH}$ & 56 & 51 & 100 \\
B. licheniformis & $\mathrm{MreB}$ & 92 & 54 & 56 \\
& $\mathrm{Mbl}$ & 53 & 97 & 51 \\
& $\mathrm{MreBH}$ & 56 & 51 & 90 \\
C. perfringens & $\mathrm{MreB}$ & 63 & 54 & 52 \\
& $\mathrm{Mbl}$ & 52 & 54 & 49 \\
& & & & \\
\hline
\end{tabular}

In contrast, expression of $m r e B^{C p}$ did not restore viability of $B$. subtilis $\Delta m r e B$ cells. No growth was observed on NA plates, nor in $\mathrm{PAB}$ medium containing $0.5 \%$ xylose. The phenotype was ameliorated in medium containing both $\mathrm{Mg}^{2+}$ and xylose (strain 4633; Fig. 3a, d): here, twisting of the cells showed that the heterologous MreB protein was expressed, but cell lysis was not prevented (strain 4633; Fig. 3b).

\section{mre $B C D^{B I}$ restores viability and rod shape of B. subtilis $\triangle$ mreBCD}

B. subtilis cells deleted for the whole $m r e B C D$ operon are only viable when grown in the presence of high $\mathrm{Mg}^{2+}$ concentrations and the osmoprotectant sucrose. Such cells have a spherical morphology, similar to $m r e C$ or $m r e D$ single mutants (Leaver \& Errington, 2005; M. Leaver, unpublished). We introduced the mreBCD operon from $B$. licheniformis into this strain background giving rise to strain 4638 and observed that expression of $m r e B C D^{B l}$ was able to restore viability, in that growth occurred on NA plates containing xylose without further additives (Fig. 4a), and that the cells displayed a rod-shaped morphology. This was true with both solid and liquid media (Fig. 4b, c). Cell shape was slightly irregular, with occasionally bent cells and some variation in cell width. Addition of $\mathrm{Mg}^{2+}$ had little effect on cell viability or morphology (Fig. 4). In the absence of inducer, the strain grew as clumps of spherical cells, the expected morphology of an mreBCD deletion mutant (M. Leaver, unpublished).

In the same way, a strain carrying an inducible copy of the $m r e B C D^{C p}$ operon was constructed (strain 4639). Just as for strain 4638 , the cells had a spherical morphology in the absence of inducer and presence of $\mathrm{Mg}^{2+}$ (Fig. 4b, c). Interestingly, expression of $m r e B C D^{C p}$ partially complemented a deletion of mreBCD in B. subtilis: in the presence of both $\mathrm{Mg}^{2+}$ and $0.5 \%$ xylose, the strain was viable and grew better than without induction of $m r e B C D^{C P}$ expression (Fig. 4a). In this strain, a high $\mathrm{Mg}^{2+}$ concentration was essential for viability: addition of xylose alone did not lead to colony formation. Unexpectedly, microscopic analysis showed that in contrast to $m r e B C D^{B l}$, expression of $m r e B C D^{C P}$ did not restore the rod shape of $B$. subtilis either on plates or in liquid medium (Fig. $4 \mathrm{~b}, \mathrm{c}$ ). 
(a)

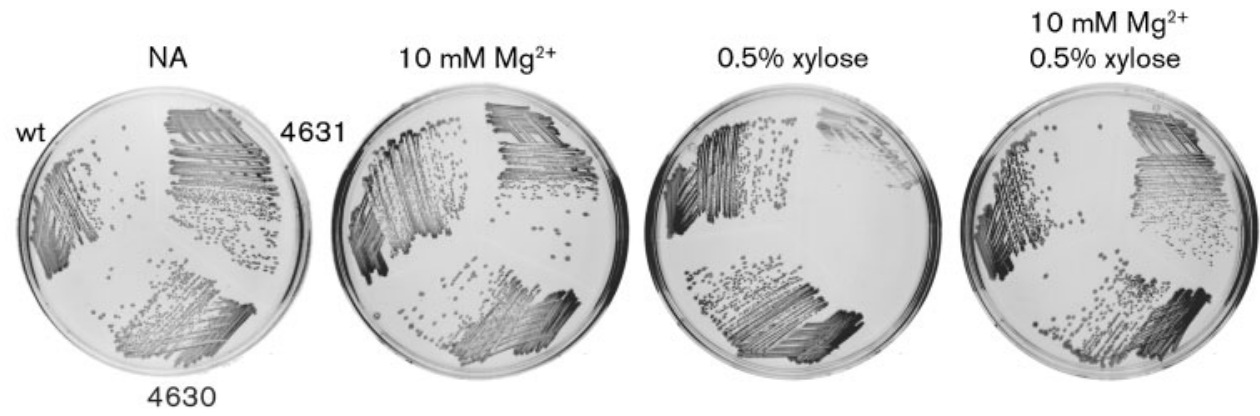

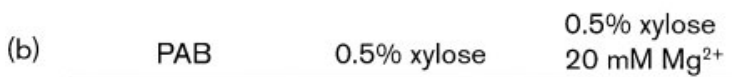

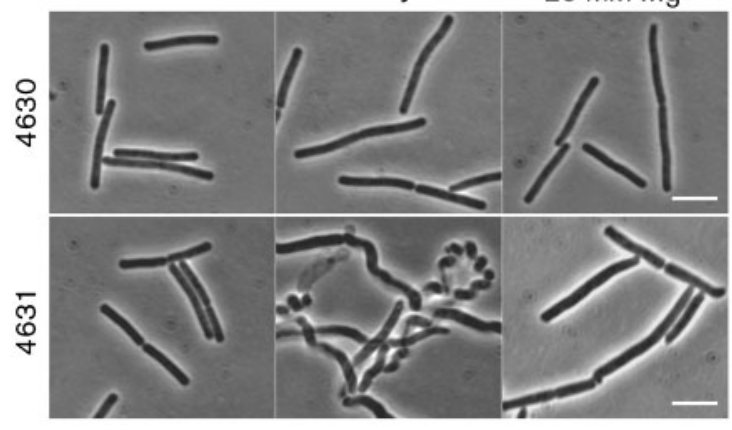

(c)

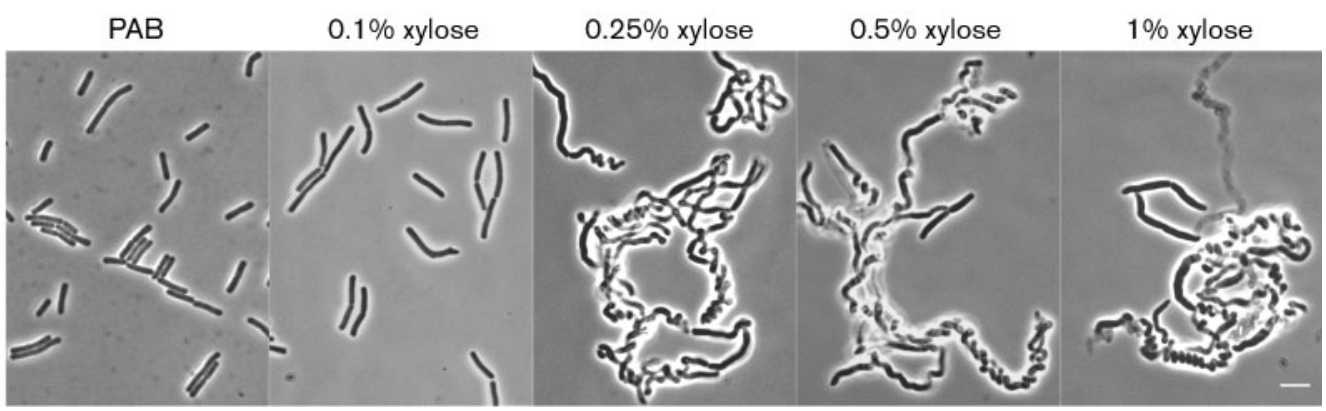

Fig. 1. Expression of $m r e B^{C p}$ has a dominant-negative effect on $B$. subtilis. (a) Growth of wild-type (wt), a strain with MreB ${ }^{B /}$ (strain 4630) and a strain with $\mathrm{MreB}^{\mathrm{C} p}$ (strain 4631) under control of a xylose inducible promoter on NA plates with additives as indicated. (b) Microscopic image of strains 4630 and 4631 grown in PAB with the supplements as indicated. (c) Phasecontrast microscopic images of strain 4631 grown in PAB with supplements as indicated. Bars, $5 \mu \mathrm{m}$.

It should be noted that expression of either heterologous $m r e B C D$ operon at least partially supported sporulation, as spores were readily found after overnight culture on NA plates (Fig. 4b, arrows).

\section{$m r e B^{B l}$ can compensate for the loss of $m b l$ in $B$. subtilis}

Previous results suggested a partial redundancy in the function of actin homologues in B. subtilis (Defeu Soufo \& Graumann, 2006; Schirner \& Errington, 2009; Kawai et al., $2009 \mathrm{~b})$. To test if $m r e B^{B l}$ was able to compensate for the loss of $m b l$, we introduced the $m b l$ deletion (Jones et al., 2001 ) into a strain with $m r e B^{B l}$ under the control of $\mathrm{P}_{x y l}$, creating strain 4634 . Interestingly, expression of $\mathrm{mreB}^{\mathrm{Bl}}$ restored the viability of the $m b l$ mutant on NA plates without supplemented $\mathrm{Mg}^{2+}$ (Fig. 5a). Also, cell shape was restored to an almost wild-type morphology, though cells were often slightly bent (Fig. 5b). In contrast, expression of $m r e B^{C p}$ did not enable growth of the $m b l$ mutant strain (strain 4635) on NA plates with inducer. Indeed, expression of this heterologous gene had a detrimental effect on $B$. subtilis $\Delta m b l$ even in the presence of high $\mathrm{Mg}^{2+}$ concentrations, leading to the formation of smaller colonies than on plates containing $\mathrm{Mg}^{2+}$ only (strain 4635; Fig. 5a). In addition, cell morphology was not rescued by expression of $m r e B^{C P}$ : the cells still twisted, swelled and lysed (Fig. 5b), similar to the unsupplemented $m b l$ mutant or to the effects of $m r e B^{C P}$ expression in a wild-type background (Fig. 1b, c). 

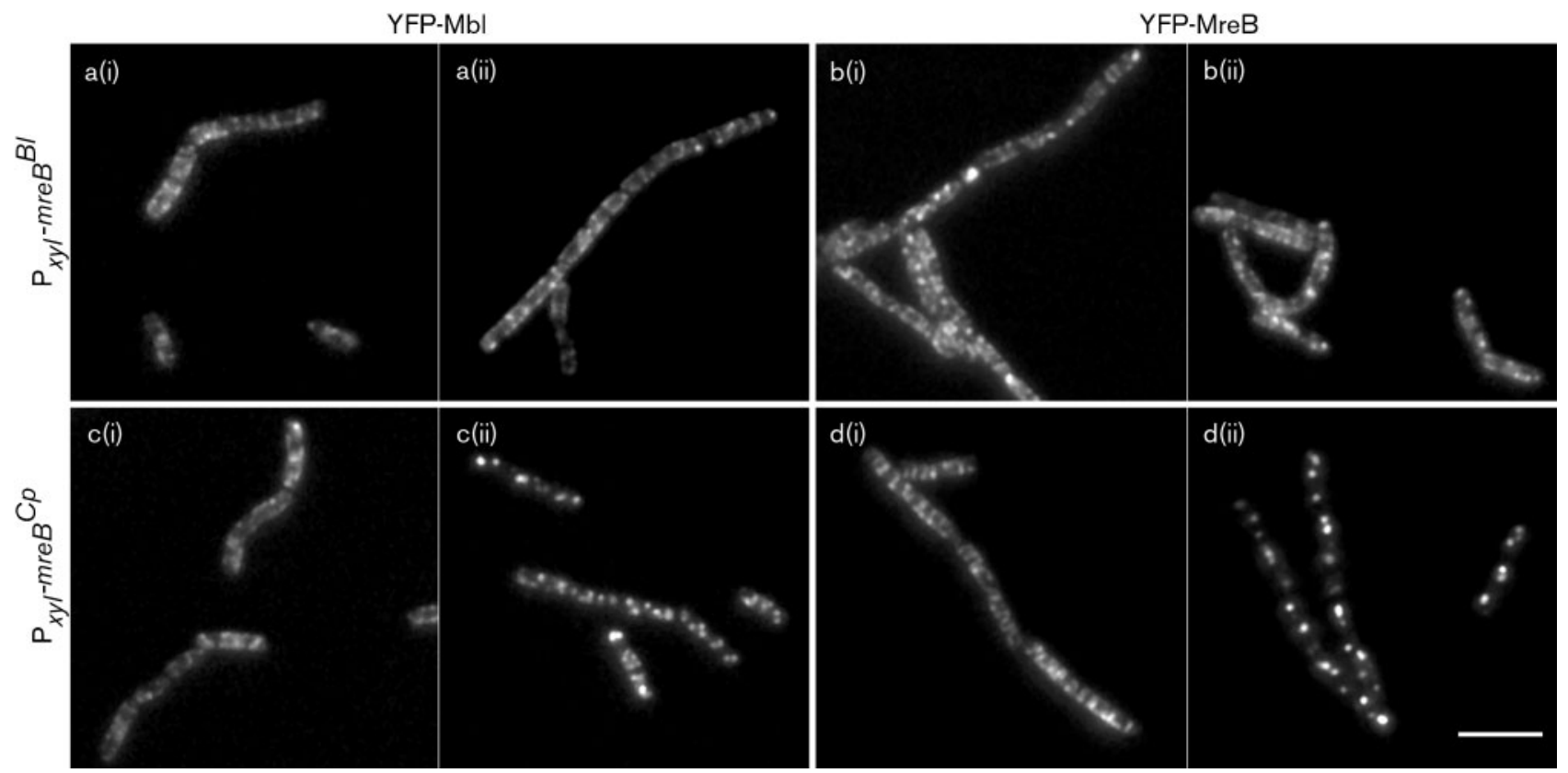

Fig. 2. Expression of $m r e B^{C p}$ disrupts the helical structures of $\mathrm{Mbl}^{B s}$ and $\mathrm{MreB}^{B s}$. (a, b) Localization of YFP-Mbl (a) and YFPMreB (b) in the absence [a(i), b(i)] and the presence [a(ii), b(ii)] of MreB $^{B l}$. (c, d) Localization of YFP-Mbl (c) and YFP-MreB (d) in the absence of $\mathrm{MreB}^{\mathrm{Cp}}[\mathrm{c}(\mathrm{i}), \mathrm{d}(\mathrm{i})]$ and when $\mathrm{MreB}^{\mathrm{Cp}}$ is expressed [c(ii), d(ii)]. Bar, $5 \mu \mathrm{m}$.

\section{The presence of one actin homologue is sufficient for determination of rod shape}

Since $m r e B^{B l}$ appeared to partially compensate for loss of either $m r e B$ or $m b l$, it was interesting to test whether it could restore growth to cells deleted for all three endogenous $m r e B$ isologues. As shown in Fig. 6, it was possible to construct such a strain (4636). The cells were slow-growing and strictly dependent on supplementation of the growth medium with $\mathrm{Mg}^{2+}$ (Fig. 6a, c). In the presence of $0.5 \%$ xylose, the cells were rod-shaped when growing on plates (Fig. 6b), but with a wide cell diameter and not uniformly shaped. In rich liquid medium such as $\mathrm{PAB}$, however, the cells did not maintain their rod shape and slowly converted to a spherical morphology (Fig. 6c). Depletion of $\mathrm{MreB}^{B l}$ under all conditions tested resulted in a more or less spherical morphology: cells tended to form clumps and lysis was frequent (Fig. 6b, c). The presence of small translucent colonies on NA plates with high $\mathrm{Mg}^{2+}$ concentrations might be due to the incomplete repression of the xylose-inducible promoter. Although $\mathrm{mreB}^{C P}$ did not complement the $B$. subtilis mreB mutant, and even had a deleterious effect when expressed in a wild-type background, it was able to confer rod-like morphology to cells with a deletion of all three endogenous $m r e B$ homologues with the $\mathrm{P}_{x y l}-m r e B^{C p}$ construct (strain 4637). Although we cannot rule out additional suppressor mutations, strain 4637 was viable and the phenotype was similar to that of strain 4636, though colonies on NA plates appeared more transparent compared to those of the strain expressing $m r e B^{B l}$ (Fig. 6a). The cells were irregularly shaped, tended to form chains and were often twisted, but became clearly rod-shaped when $m r e B^{C P}$ was expressed. In the absence of xylose, the cells became spherical and often lysed (Fig. 6b, c). Elevated $\mathrm{Mg}^{2+}$ concentrations were essential for viability (Fig. 6a, c). Nevertheless, these results showed that B. subtilis viability and cell elongation could be substantially rescued by expression of a single $m r e B$ gene only $63 \%$ identical to that of the wild-type.

\section{DISCUSSION}

\section{$M B^{C p}$ interferes with the $B$. subtilis actin cytoskeleton}

Surprisingly, the overexpression of $m r e B^{C P}$ had a dominant-negative effect on viability of wild-type B. subtilis, whilst overexpression of $m r e B^{B l}$ had no effect on cell shape or viability. The morphology of cells expressing $m r e B^{C p}$ was highly reminiscent of that of $m b l$ mutants: in both cases, the cells twist, swell and lyse, and they are not viable unless the medium is supplemented with high concentrations of $\mathrm{Mg}^{2+}$ (Abhayawardhane \& Stewart, 1995; Jones et al., 2001; Schirner \& Errington, 2009). The delocalization of $\mathrm{Mbl}$ and $\mathrm{MreB}$ in a strain expressing $m r e B^{C P}$ suggests that this heterologous MreB interferes with the polymerization of $B$. subtilis actin homologues, which have been shown to form a single helical structure and therefore probably interact with each other or a common target protein 
(a)
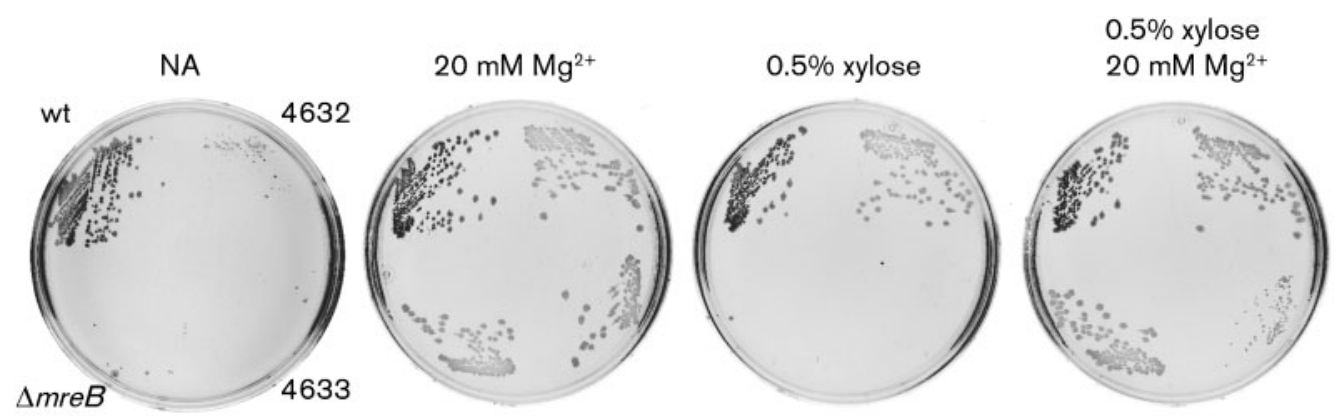

(b)

PAB

PAB $20 \mathrm{mM} \mathrm{Mg}^{2+}$

$0.5 \%$ xylose

$0.5 \%$ xylose

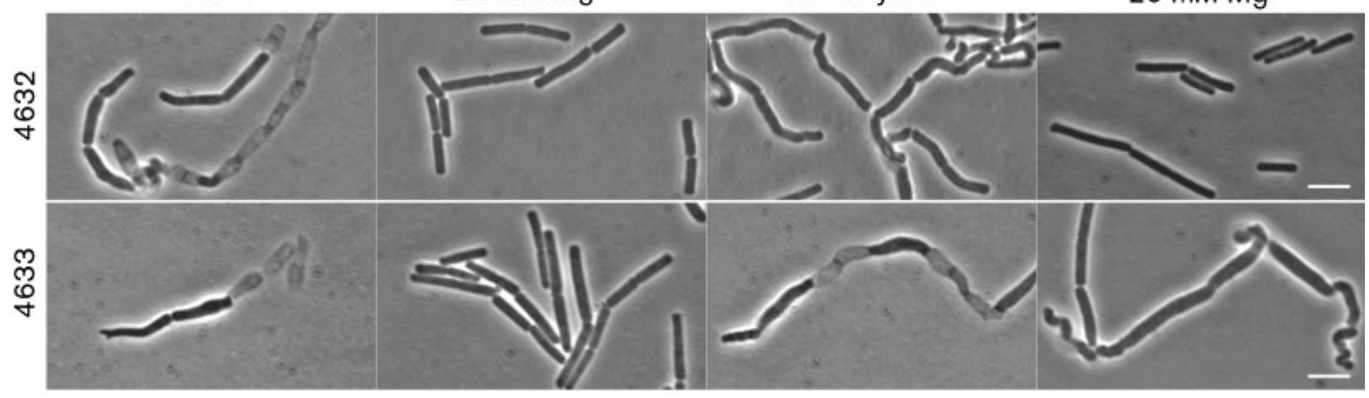

(c)

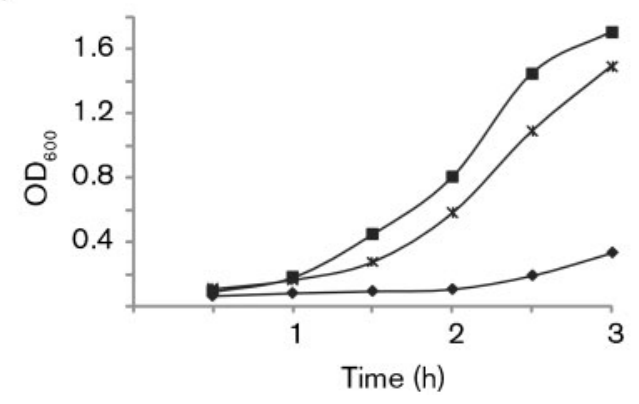

(d)

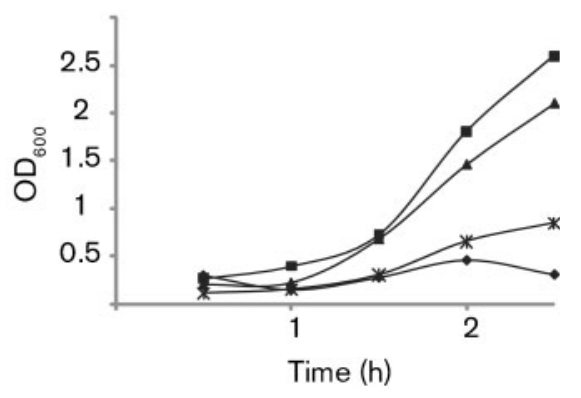

Fig. 3. $m r e B^{B l}$ restores the viability of a $B$. subtilis mreB mutant. (a) Growth of wild-type, mre $B$ mutant, an mre $B$ mutant strain carrying $m r e B^{B I}$ (strain 4632) or $m r e B^{C p}$ (strain 4633) under the control of $\mathrm{P}_{x y l}$ on NA plates with supplements as indicated. (b) Phase-contrast microscopy images showing morphology of strains 4632 and 4633 when grown in PAB medium with supplements as indicated at $37^{\circ} \mathrm{C}$. Bars, $5 \mu \mathrm{m}$. (c, d) Growth curves of strain 4632 (c) and strain 4633 (d) in PAB (॰), PAB supplemented with $20 \mathrm{mM} \mathrm{Mg}^{2+}(\boldsymbol{\square}), 0.5 \%$ xylose $(*)$ or both $20 \mathrm{mM} \mathrm{Mg}^{2+}$ and $0.5 \%$ xylose $(\boldsymbol{\Delta})$ at $37^{\circ} \mathrm{C}$.

(Carballido-López et al., 2006). Disruption of the helical structure leads to cell shape defects and ultimately loss of viability, possibly due to an imbalance of processes involved in cell wall synthesis and turnover which are regulated in subtly differentiated ways by the various isologues.

\section{The MreBCD complex alone is not sufficient for determination of rod shape}

$\mathrm{MreC}$ and MreD are important for rod-shape determination in B. subtilis and E. coli. They probably form a complex with MreB, and together they are involved in orchestrating lateral cell wall synthesis (Defeu Soufo \&
Graumann, 2005; Kruse et al., 2005; Leaver \& Errington, 2005; Wachi et al., 1989). The results presented here show that the MreBCD homologues of B. licheniformis are able to restore rod shape and viability in $B$. subtilis $\triangle m r e B C D$, indicating that the similarity of $\mathrm{MreC}$ and $\mathrm{MreD}$ from $B$. licheniformis to the B. subtilis proteins (71 and $63 \%$, respectively) is high enough to substitute for the endogenous proteins. However, expression of $m r e B C D^{C P}$ failed to complement the mreBCD mutant, even though the presence of $\mathrm{MreB}^{C P}$ as the only MreB protein is enough for determination of rod shape. It is possible that the MreBCD ${ }^{C p}$ complex fails to interact with, or even inhibits, other proteins involved in synthesis of the cylindrical part of the cell wall, such as RodA, RodZ or penicillin-binding 
(a)

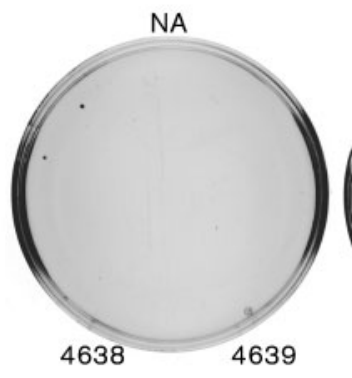

(b)

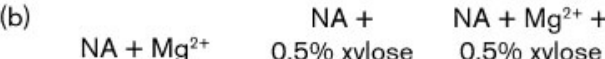
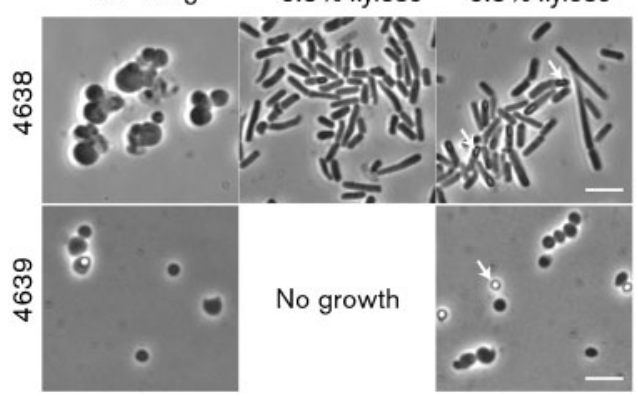

$20 \mathrm{mM} \mathrm{Mg}^{2+}$
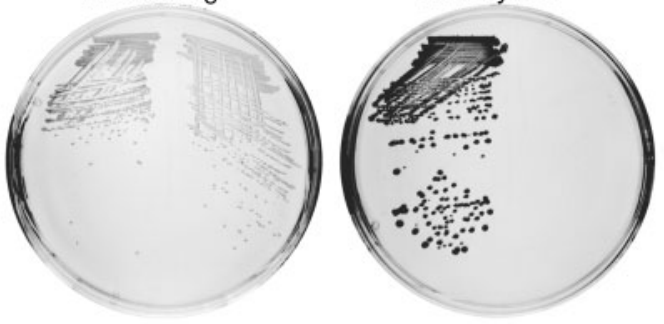

$0.5 \%$ xylose

$20 \mathrm{mM} \mathrm{Mg}^{2+}$

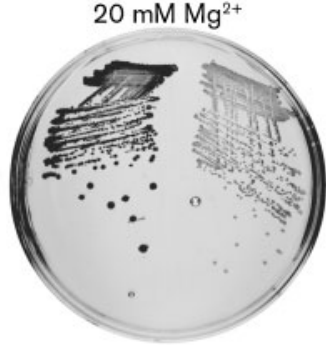

(c)

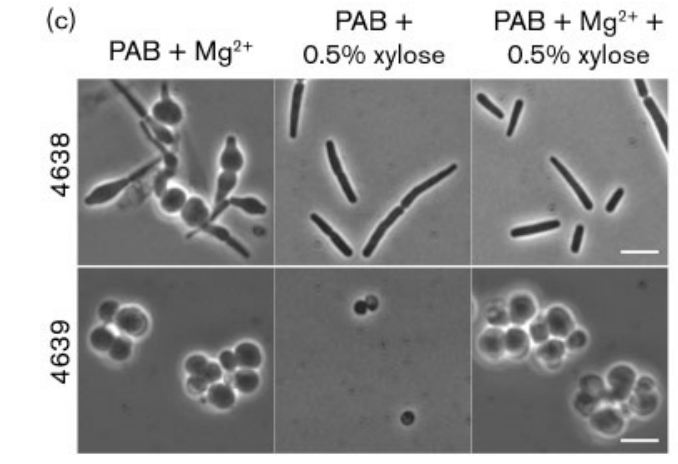

Fig. 4. Viability of $B$. subtilis $\triangle m r e B C D$ expressing $m r e B C D^{B /}$. (a) Growth of $B$. subtilis $\triangle m r e B C D$ carrying either $m r e B C D^{B \prime}$ (strain 4638) or mreBCD ${ }^{C p}$ (4639) on NA plates containing supplements as indicated. (b, c) Phase-contrast microscopy images showing morphology of strains 4638 and 4639 when grown on NA plates (b) or in liquid PAB medium (c) containing $\mathrm{Mg}^{2+}$ and xylose as indicated. Bars, $5 \mu \mathrm{m}$.

(a)

NA

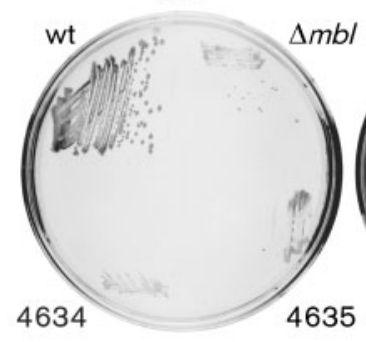

4635

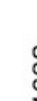

Fig. 5. Expression of $m r e B^{B l}$ confers viability to $B$. subtilis $\Delta m b l$. (a) $B$. subtilis wild-type, $m b /$ mutant, and $m b /$ mutant strains carrying an inducible copy of $m r e B^{B /}(4634)$ or $m r e B^{C p}$ (4635) grown on NA plates with supplements as indicated. (b) Phase-contrast microscopic images showing cell morphology of strains 4634 and 4635 grown in PAB medium at $37{ }^{\circ} \mathrm{C}$ with additives as indicated. Bars, $5 \mu$ m. 
(a)

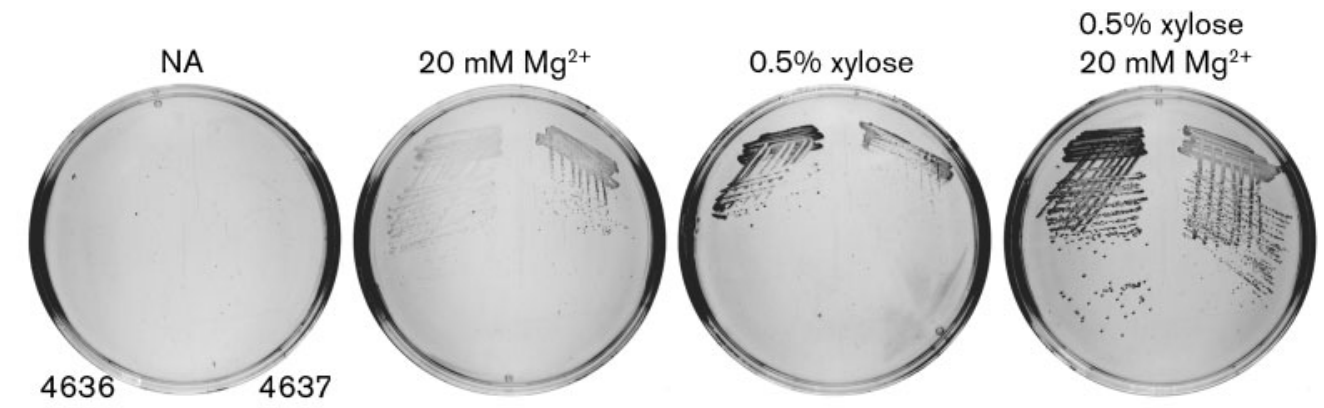

$\begin{array}{lll} & \mathrm{NA}+ & \mathrm{NA}+\mathrm{Mg}^{2+}+ \\ \text { (b) } \quad \mathrm{NA}+\mathrm{Mg}^{2+} & 0.5 \% \text { xylose } & 0.5 \% \text { xylose }\end{array}$

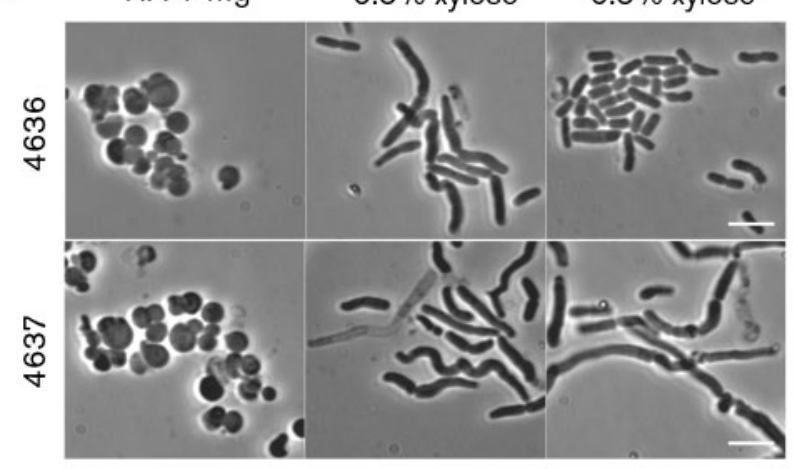

(c)

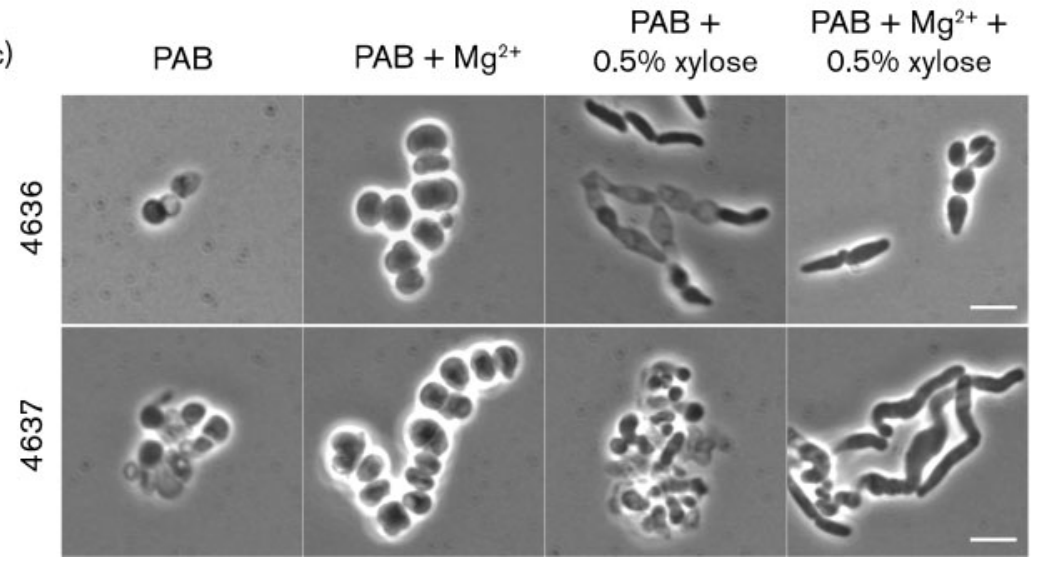

Fig. 6. Viability of a $B$. subtilis strain expressing only a single heterologous MreB protein. (a) Growth of $B$. subtilis $\Delta m r e B \Delta m b /$ $\Delta m r e B H$ with an inducible copy of $m r e B^{B \prime}(4636)$ or $m r e B^{C p}$ (4637) on NA plates with additives as indicated. (b, c) Cell shape of strains 4636 and 4637 when grown on NA plates (b) or in PAB medium (c) at $37{ }^{\circ} \mathrm{C}$ under conditions as indicated. Bars, $5 \mu \mathrm{m}$.

proteins (Alyahya et al., 2009; Bendezu et al., 2009; Rogers \& Taylor, 1978; Shiomi et al., 2008; Wei et al., 2003), resulting in the formation of spherical cells. The membrane proteins $\mathrm{MreC}$ and $\mathrm{MreD}$ probably have a function in connecting the intracellular actin scaffold to the extracellular cell wall synthetic machinery (Leaver \& Errington, 2005; van den Ent et al., 2006). Since these proteins are only 21 and $16 \%$ identical, respectively, to the B. subtilis homologues, $\mathrm{MreC}^{C P}$ and $\mathrm{MreD}^{C P}$ are probably too divergent to fulfil their functions in B. subtilis. Because MreBCD $^{C P}$ should be able to form a functional complex in B. subtilis, this result suggests that the formation of a functional MreBCD complex is not enough for determination of rod shape, but interactions with other morphogenic proteins are required. However, we cannot exclude the possibility that one or both of $\mathrm{MreC}^{C p}$ or $\mathrm{MreD}^{C P}$ do not accumulate sufficiently for reasons of gene expression levels, activity or stability in B. subtilis. 


\section{Overlapping functions of actin homologues}

Although B. subtilis strains with single deletions of $m r e B$ homologues can be constructed and are viable in the presence of high $\mathrm{Mg}^{2+}$ concentrations, the only viable double mutant has deletions of $\mathrm{mbl}$ and $\mathrm{mreBH}$ (Defeu Soufo \& Graumann, 2006; Kawai et al., 2009b). Interestingly, overexpression of $m r e B^{B l}$ not only restored viability of a $B$. subtilis mreB mutant, it also rescued an $m b l$ deletion strain, supporting the idea of a functional overlap between $m r e B$ and $m b l$. Subsequently, strains carrying deletions of all three endogenous $m r e B$ homologues were constructed in the presence of $m r e B^{B l}$ or $m r e B^{C P}$ under the control of $\mathrm{P}_{x y l}$. Although these cells were by no means wildtype in phenotype, they clearly retained the ability to elongate and became distinctly rod-like. Thus, a single MreB homologue, only $63 \%$ identical to the endogenous MreB, is sufficient for determination of longitudinal growth in B. subtilis. Therefore, it seems likely that there is significant functional redundancy between MreB isologues.

Given the apparent functional complementation of an $m r e B$ isologue triple mutant, it was surprising that $m r e B^{C P}$ had a deleterious effect when expressed in a wild-type background or in $m r e B$ or $m b l$ single mutant strains (Figs 1, 3 and 5, respectively). Based on the results shown in Fig. 2, we suggest that this dominant-negative phenotype is due to interference in assembly of the endogenous MreB isologues into filaments, leading to disturbed cell growth. The interference effect can apparently be manifested through either MreB or Mbl. The partial complementation in a triple mreB null background suggests that the $\mathrm{MreB}^{C P}$ protein can nevertheless interact productively with the wall synthetic machinery. We hope to use $m r e B^{C P}$ as a tool to help identify and dissect the sites on MreB isologues involved in self-interaction, leading to filament assembly, versus those involved in interactions with the effector proteins of cell wall synthesis.

\section{ACKNOWLEDGEMENTS}

This work was supported by a grant from the UK Biotechnology and Biological Sciences Research Council. We thank Mark Leaver for strain 4334, Leendert Hamoen for oligonucleotides amy1 and amy2, Yoshi Kawai for YFP-Mbl and YFP-MreB constructs, and Alex Formstone for helpful discussions.

\section{REFERENCES}

Abhayawardhane, Y. \& Stewart, G. C. (1995). Bacillus subtilis possesses a second determinant with extensive sequence similarity to the Escherichia coli mreB morphogene. J Bacteriol 177, 765-773.

Altschul, S. F., Gish, W., Miller, W., Myers, E. W. \& Lipman, D. J. (1990). Basic local alignment search tool. J Mol Biol 215, 403-410.

Alyahya, S. A., Alexander, R., Costa, T., Henriques, A. O., Emonet, T. \& Jacobs-Wagner, C. (2009). RodZ, a component of the bacterial core morphogenic apparatus. Proc Natl Acad Sci U S A 106, 12391244.
Anagnostopoulos, C. \& Spizizen, J. (1961). Requirements for transformation in Bacillus subtilis. J Bacteriol 81, 741-746.

Bendezu, F. O., Hale, C. A., Bernhardt, T. G. \& de Boer, P. A. (2009). $\operatorname{RodZ}(\mathrm{YfgA}$ ) is required for proper assembly of the MreB actin cytoskeleton and cell shape in E. coli. EMBO J 28, 193-204.

Bork, P., Sander, C. \& Valencia, A. (1992). An ATPase domain common to prokaryotic cell cycle proteins, sugar kinases, actin, and hsp70 heat shock proteins. Proc Natl Acad Sci U S A 89, 7290-7294.

Carballido-Lopez, R. (2006a). Orchestrating bacterial cell morphogenesis. Mol Microbiol 60, 815-819.

Carballido-López, R. (2006b). The bacterial actin-like cytoskeleton. Microbiol Mol Biol Rev 70, 888-909.

Carballido-López, R., Formstone, A., Li, Y., Ehrlich, S. D., Noirot, P. \& Errington, J. (2006). Actin homolog MreBH governs cell morphogenesis by localization of the cell wall hydrolase LytE. Dev Cell 11, 399-409.

Daniel, R. A. \& Errington, J. (2003). Control of cell morphogenesis in bacteria: two distinct ways to make a rod-shaped cell. Cell 113, 767776.

Defeu Soufo, H. J. \& Graumann, P. L. (2005). Bacillus subtilis actinlike protein $\mathrm{MreB}$ influences the positioning of the replication machinery and requires membrane proteins $\mathrm{MreC} / \mathrm{D}$ and other actinlike proteins for proper localization. BMC Cell Biol 6, 10.

Defeu Soufo, H. J. \& Graumann, P. L. (2006). Dynamic localization and interaction with other Bacillus subtilis actin-like proteins are important for the function of MreB. Mol Microbiol 62, 1340-1356.

Figge, R. M., Divakaruni, A. V. \& Gober, J. W. (2004). MreB, the cell shape-determining bacterial actin homologue, co-ordinates cell wall morphogenesis in Caulobacter crescentus. Mol Microbiol 51, 1321-1332.

Formstone, A. \& Errington, J. (2005). A magnesium-dependent mreB null mutant: implications for the role of mreB in Bacillus subtilis. Mol Microbiol 55, 1646-1657.

Glaser, P., Sharpe, M. E., Raether, B., Perego, M., Ohlsen, K. \& Errington, J. (1997). Dynamic, mitotic-like behavior of a bacterial protein required for accurate chromosome partitioning. Genes Dev 11, 1160-1168.

Hayhurst, E. J., Kailas, L., Hobbs, J. K. \& Foster, S. J. (2008). Cell wall peptidoglycan architecture in Bacillus subtilis. Proc Natl Acad Sci U S A 105, 14603-14608.

Jenkinson, H. F. (1983). Altered arrangement of proteins in the spore coat of a germination mutant of Bacillus subtilis. J Gen Microbiol 129, 1945-1958.

Jones, L. J., Carballido-López, R. \& Errington, J. (2001). Control of cell shape in bacteria: helical, actin-like filaments in Bacillus subtilis. Cell 104, 913-922.

Kawai, Y., Daniel, R. A. \& Errington, J. (2009a). Regulation of cell wall morphogenesis in Bacillus subtilis by recruitment of PBP1 to the MreB helix. Mol Microbiol 71, 1131-1144.

Kawai, Y., Asai, K. \& Errington, J. (2009b). Partial functional redundancy of $\mathrm{MreB}$ isoforms, $\mathrm{MreB}, \mathrm{Mbl}$ and $\mathrm{MreBH}$, in cell morphogenesis of Bacillus subtilis. Mol Microbiol 73, 719-731.

Kruse, T., Bork-Jensen, J. \& Gerdes, K. (2005). The morphogenetic MreBCD proteins of Escherichia coli form an essential membranebound complex. Mol Microbiol 55, 78-89.

Larkin, M. A., Blackshields, G., Brown, N. P., Chenna, R., McGettigan, P. A., McWilliam, H., Valentin, F., Wallace, I. M., Wilm, A. \& other authors (2007). CLUSTAL W and CLUSTAL X version 2.0. Bioinformatics 23, 2947-2948.

Leaver, M. \& Errington, J. (2005). Roles for MreC and MreD proteins in helical growth of the cylindrical cell wall in Bacillus subtilis. Mol Microbiol 57, 1196-1209. 
Lee, J.-C. \& Stewart, G. C. (2003). Essential nature of the mreC determinant of Bacillus subtilis. J Bacteriol 185, 4490-4498.

Nicholson, W. L. \& Setlow, P. (1990). Sporulation, germination and outgrowth. In Molecular Biological Methods for Bacillus, pp. 391-450. Edited by C. R. Harwood \& S. M. Cutting. New York: Wiley.

Rogers, H. J. \& Taylor, C. (1978). Autolysins and shape change in rodA mutants of Bacillus subtilis. J Bacteriol 135, 1032-1042.

Rogers, H. J., McConnell, M. \& Burdett, I. D. (1970). The isolation and characterization of mutants of Bacillus subtilis and Bacillus licheniformis with disturbed morphology and cell division. J Gen Microbiol 61, 155-171.

Sambrook, J., Fritsch, E. F. \& Maniatis, T. (1989). Molecular Cloning: a Laboratory Manual. Cold Spring Harbor, NY: Cold Spring Harbor Laboratory.

Schirner, K. \& Errington, J. (2009). Cell wall regulator $\sigma^{\mathrm{I}}$ specifically suppresses the lethal phenotype of mbl mutants in B. subtilis. $J$ Bacteriol 191, 1404-1413.

Shiomi, D., Sakai, M. \& Niki, H. (2008). Determination of bacterial rod shape by a novel cytoskeletal membrane protein. EMBO J 27, 30813091 .
Stewart, G. C. (2005). Taking shape: control of bacterial cell wall biosynthesis. Mol Microbiol 57, 1177-1181.

Tiyanont, K., Doan, T., Lazarus, M. B., Fang, X., Rudner, D. Z. \& Walker, S. (2006). Imaging peptidoglycan biosynthesis in Bacillus subtilis with fluorescent antibiotics. Proc Natl Acad Sci U S A 103, 11033-11038.

van den Ent, F., Amos, L. A. \& Löwe, J. (2001). Prokaryotic origin of the actin cytoskeleton. Nature 413, 39-44.

van den Ent, F., Leaver, M., Bendezu, F., Errington, J., de Boer, P. \& Löwe, J. (2006). Dimeric structure of the cell shape protein MreC and its functional implications. Mol Microbiol 62, 1631-1642.

Wachi, M., Doi, M., Okada, Y. \& Matsuhashi, M. (1989). New mre genes $m r e C$ and $m r e D$, responsible for formation of the rod shape of Escherichia coli cells. J Bacteriol 171, 6511-6516.

Wei, Y., Havasy, T., McPherson, D. C. \& Popham, D. L. (2003). Rod shape determination by the Bacillus subtilis class B penicillinbinding proteins encoded by $p b p A$ and $p b p H$. J Bacteriol 185, 47174726.

Edited by: S. J. Foster 